\title{
“SAY BOO TO THE FLU!” InTROdUCTION OF THE Seasonal Influenza Peer Vaccination Programme in THE EMERGENCY DePARTMENT
}

\author{
AND AMAU
}

\author{
Authors: Lisa Donaghy, MSc, PGDip, BSc, RGN, and Patrick Martin, MSc, HDip, PGDip, RGN, RNP, Dublin, Ireland \\ Section Editors: Cindy Lefton, PhD, RN, and Jennifer Williams, PhD, RN, ACNS-BC, CCRN, CNS
}

$\mathrm{T}$

he Seasonal Influenza Peer Vaccination Programme was developed by the Republic of Ireland Health Service Executive (HSE) ${ }^{1}$ with the aim of increasing the number of health care workers receiving annual flu vaccinations. Supporting these efforts, various studies illustrated that increasing health care staff vaccination rates decreased patient illness and death, ${ }^{2}$ while other research reported a $40 \%$ reduction of influenza-related deaths in hospitals with higher rates of health care workers' influenza vaccinations. ${ }^{3}$ Flu vaccination recommendations from The World Health Organization (WHO) include a target uptake of $75 \%$ in health care staff and people age 65 and older. ${ }^{3}$

In accordance with HSE national guidelines that health care organizations achieve and/or surpass the minimum threshold goal of $40 \%$ of employees receiving the flu vaccine, ${ }^{4}$ and recognizing that Peer-to-Peer Influenza Vaccination Programmes are an essential aspect of the overall infection prevention and control arrangements in our health care setting, Connolly Hospital In Dublin, Ireland, initiated the Peer Vaccination Programme in the Emergency Department and Acute Medical Assessment Unit (AMAU) during the 2016-2017 flu season. The challenges facing successful ED and AMAU programme implementation included the large volume of interdisciplinary staff serving the ED area, a vast cohort of undifferentiated complex patients at risk for increased complications if exposed to influenza, and an elevated

\footnotetext{
Lisa Donaghy is Clinical Facilitator, Emergency Department, Acute Medical Assessment Unit, Connolly Hospital, Dublin, Ireland

Patrick Martin is Registered Advanced Nurse Practitioner in Emergency, Connolly Hospital, Dublin, Ireland

The opinions expressed here are those of the respondents and should not be construed as the official position of the institution, the ENA, or the journal. For correspondence, write: Patrick Martin, MSc, Hdip, PGDip, RGN, RNP, Emergency Department, Connolly Hospital, Blanchardstown, Dublin 15, Ireland; E-mail: patrick.martin@hse.ie.

J Emerg Nurs 2018;44:418-20.

0099-1767

Copyright ( 2018 Emergency Nurses Association. Published by Elsevier Inc. All rights reserved.

https://doi.org/10.1016/j.jen.2018.04.008
}

risk of exposure to the influenza virus for all members of the health care team.

Similar to programs in other countries, the Peer-to-Peer Vaccination Programme vaccinates health care workers against the influenza viruses identified as those most likely to circulate in the upcoming season. ${ }^{5}$ Although recognizing that frontline staff have a duty of care to protect their patients and colleagues, receiving the flu vaccine is not a mandatory requirement for health care worker employment in Ireland.

\section{Peer-to-Peer Vaccinator Criteria}

Two peer vaccinators were trained for the emergency department/AMAU for 2016. Vaccinator requirements included an $\mathrm{RN}$ and/or midwife on the live register of the Nursing and Midwifery Board of Ireland (NMBI), basic life support training within the last 2 years, anaphylaxis training and updates within 2 years, and competency in injection technique. Furthermore, the vaccinator candidates were required to attend the Seasonal Influenza Peer Vaccination Programme Training Day.

\section{Peer-to-Peer Vaccine Clinics}

Information about upcoming clinics and the availability of the programme were communicated during interdisciplinary daily huddles in the emergency department and AMAU. Posters were distributed to inform staff to avail of the influenza vaccine, highlight the peer vaccinator names, and illustrate "Myths and Facts." These posters provided essential information to health care workers so they could make informed decisions regarding their participation in the programme. Both the peer vaccinators worked within the emergency department and AMAU 7 days a week from 8:00 am to $8: 30 \mathrm{pm}$. The long hours and successive workdays allowed staff from all shifts during their work time to access the clinic and provided an opportunity for the vaccinators to address queries about the programme and/or vaccine. Despite this length of time, clinic accessibility was 
dependent upon ED workload, vaccinator availability, and demand. Although 111 interdisciplinary staff members in the emergency department and AMAU were eligible to participate in the Peer Vaccination Programme, some employees were on vacation or leave during this period. Therefore, 97 employees were eligible to participate in this programme, and 44 staff members were vaccinated.

\section{Adverse Reaction}

An anaphylaxis reaction occurred in 1 health care worker following the administration of inactivated influenza (Split Virion) BP $0.5 \mathrm{~mL}$ administered intramuscularly (IM). The staff member started experiencing a sudden onset of dyspnea, bronchospasm, erythema, pruritus, and tachypnoea approximately 4 minutes after the injection. Anaphylaxis treatment consisting of IM adrenaline $0.5 \mathrm{mg}$, intravenous (IV) chlorphenamine $10 \mathrm{mg}$, IV hydrocortisone $200 \mathrm{mg}$, and salbutamol nebulizer $2.5 \mathrm{mg}$ with oxygen therapy were administered immediately by the peer vaccinators, and expert help was sought. The reaction lasted approximately 45 minutes, and symptoms reappeared 4 hours later. Two years before this vaccination, the health care worker had received the influenza vaccine with no adverse reactions. An overnight hospital admission for relief of symptoms and observation was required. However, no fatal outcomes occurred.

\section{Peer Flu Vaccination Campaign Data}

An anonymous survey was circulated to all 44 staff who received the vaccine. The response rate was $84 \%(\mathrm{~N}=37)$.

Fifty-seven percent of the respondents $(\mathrm{N}=21)$ indicated that, in the previous year (2015), they had received the flu vaccine. Forty-three percent of the

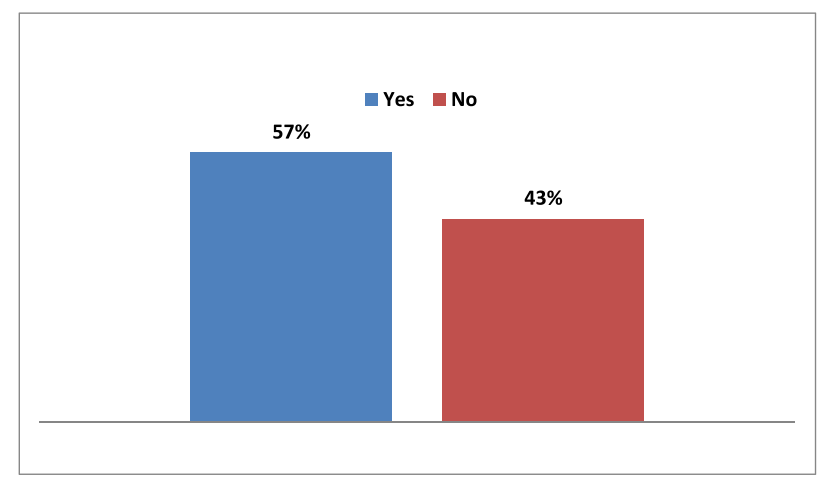

FIGURE 1

Did you receive the flue vaccine in 2015?

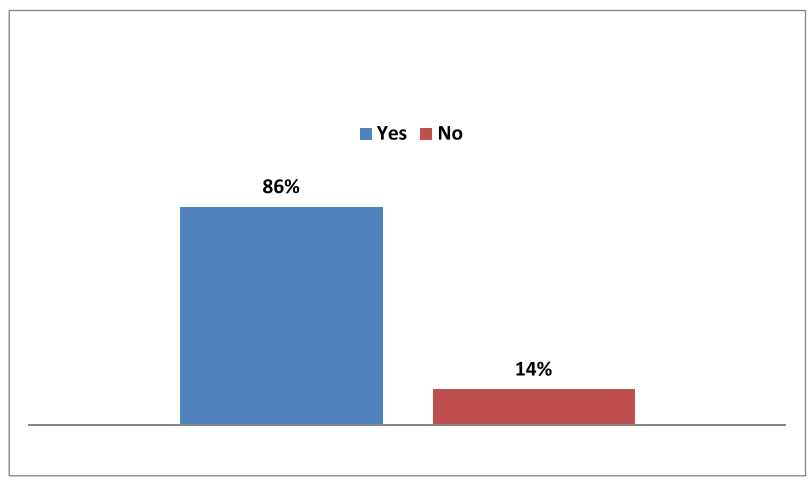

FIGURE 2

Did the Peer Vaccination Campaign encourage you to receive the flue vaccine in 2016 ?

respondents $(\mathrm{N}=16)$ stated that they did not receive a flu vaccination in 2015 (Figure 1).

In regard to the positive influence of the Peer Vaccination Campaign, $86 \%(\mathrm{~N}=32)$ of the participants indicated that this programme influenced their effort to get vaccinated, whereas $14 \%(\mathrm{~N}=5)$ of the participants were not motivated by the programme to obtain their flu vaccines (Figure 2).

In regard to future impact, $95 \%(\mathrm{~N}=35)$ of the participants who received the vaccine stated that they would get vaccinated the following year, whereas 5\% $(\mathrm{N}=2)$ indicated that they would not obtain a flu vaccination the following year (Figure 3 ).

One hundred percent of the participants indicated the peer vaccination campaign was helpful and should occur the following year (Figure 4).

\section{Conclusion}

Upon implementation of the Peer-to-Peer Vaccination Clinic, we were able to encourage an additional $16 \mathrm{ED} /$ AMAU staff members who did not receive the flu vaccine in

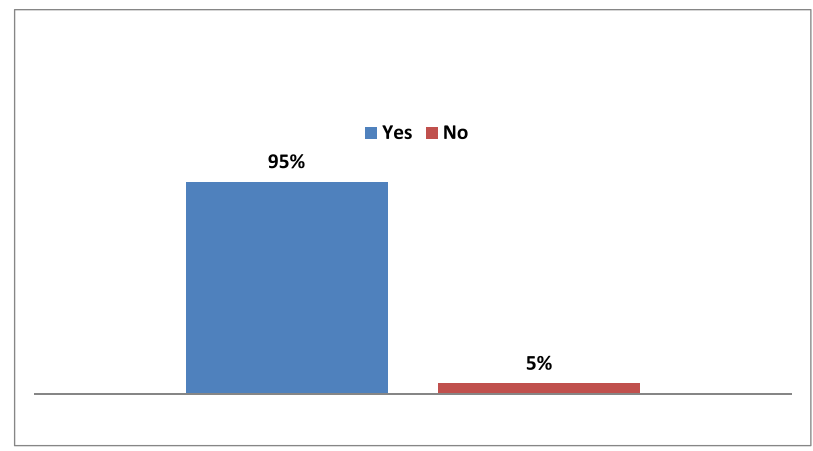

FIGURE 3

Would you get the flu vaccine next year (2017)? 


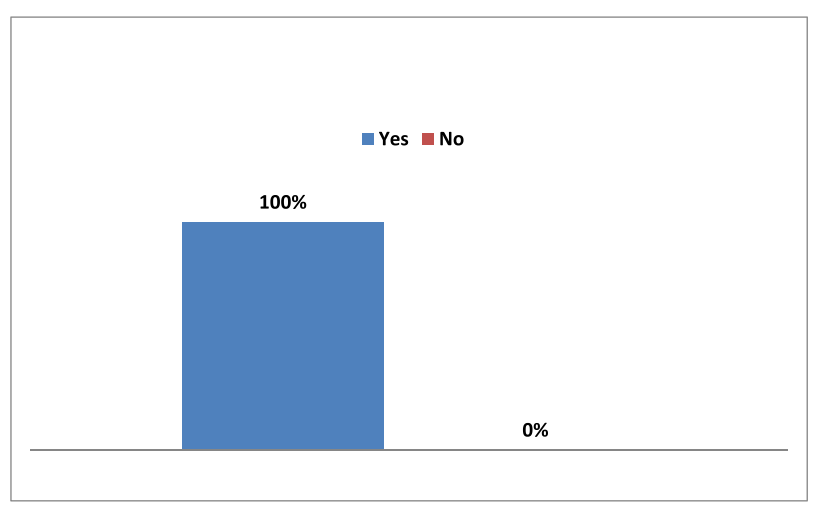

FIGURE 4

Would you recommend the peer vaccination campaign to continue next year?

2015-2016 to take up the vaccine during the 2016-2017 campaign. Overall, Connolly Hospital experienced a 2.8\% increase in the number of vaccinated employees across the organization, amounting to $34.7 \%$ of the hospital-wide staff receiving the influenza vaccine. Despite this increase, we did not meet the HSE 40\% minimum threshold. In the forthcoming years, our goal is the implementation of a widespread programme to increase the uptake of the influenza vaccine, and Connolly Hospital managers will continue to facilitate and support those willing to participate in the Peer Vaccination Programme.

\section{REFERENCES}

1. Health Service Executive. Seasonal Influenza Peer Vaccination Programme, Guidelines for Staff. National Immunisation Office. Dublin, Ireland; 2016.

2. World Health Organization (WHO). Influenza (Seasonal): Fact Sheet. http:// www.who.int/mediacentre/factsheets/fs211/en/. Accessed September 10, 2017.

3. Carman WF, Elder AG, Wallace LA, et al. Effects of influenza vaccination of healthcare workers on mortality of elderly people in long-term care: a randomised controlled trial. Lancet. 2000;355(9198):93-97.

4. National Health Service (NHS) England. The National Flu Immunisation Programme 2016/17;2016. http://hcp.influvac.co.uk/PDFs/ Annual_flu_letter_2016_2017.pdf. Accessed September 15, 2017.

5. Health Service Executive. Continuous Professional Education Programme for Registered Nurses and Midwives to administer Inactivated Influenza Vaccine (Split Virion) BP Under the Medicine Protocol to Nurses, Midwives and Healthcare Workers; 2017. http://www.hse.ie/eng/health/ Immunisation/hcpinfo/fluinfo/MedProtocolFlu2017.pdf. Accessed on September 15, 2017.

Submissions to this column are encouraged and may be sent to Cindy Lefton, PhD, RN

clefton@q4solutions.com or Jennifer Williams, PhD, RN, ACNS-BC, CCRN, CNS jawilliamscns@gmail.com 